Interdisciplinary Studies of Complex Systems

No. 14 (2019) 35-52

(C) A. Leiko, A. Derepa, I. Averichev, O. Kocharian, Y. Starovoit

https://doi.org/10.31392/iscs.2019.14.035

\title{
MUTUAL INFLUENCE OF ELECTRICAL, MECHANICAL AND ACOUSTIC FIELDS UNDER THE RADIATION OF SOUND BY CIRCULAR SYSTEMS WITH SCREEN, FORMED FROM CYLINDRICAL PIEZOCERAMIC RADIATORS
}

\author{
Aleksandr Leiko, Anatolii Derepa ${ }^{2}$, Ilia Averichev, \\ Oksana Kocharian, Yaroslav Starovoit ${ }^{4}$
}

\begin{abstract}
With the help of coupled fields method in multiply connected regions, we obtained analytical relations describing the physical fields of cylindrical piezoceramic radiators with circumferential polarization, which are part of cylindrical systems with a screen in the internal cavity. Based on the obtained relationships, quantitative estimates of the parameters of the electric, mechanical, and acoustic fields of the radiatos are performed when operating in circular systems with a screen, the analysis of which revealed the degree of influence of the fields on each other.
\end{abstract}

Keywords: physical fields; circular screen system; piezoceramic cylindrical radiator; circular polarization

\section{Introduction}

One of the main parts of complex echolocation systems is their radiation system. At the same time, sound location systems differ in two features that are characteristic only for them, namely, the conversion of one type of energy into another and the formation of acoustic energy in the surrounding space [1]. Each of these features has a number of physical properties, without which, when calculating the parameters of the systems, there are significant differences between their calculated and actual values. At present, when constructing sound emitting systems, the main element for converting electrical energy first to mechanical and then to acoustic is piezoceramic radiators. They are characterized by a deep interconnection between electric, mechanical and acoustic fields [2], influencing energy conversion processes [3-5]. During the formation of acoustic fields in the external space of the systems, interaction between the elements of an acoustic field occurs between their elements. This interaction is

\footnotetext{
${ }^{1}$ National Technical University of Ukraine "Igor Sikorsky Kyiv Polytechnic Institute" Peremohy Ave., 37, Kyiv 03056, Ukraine

${ }^{2}$ State University of Infrastructure and Technology, Kirilovskaya Str., 9, Kyiv 02000, Ukraine

${ }^{3}$ Central Research Institute of Armament and Military Equipment of the Armed Forces of Ukraine, Povitroflotskyi Ave., 28/69, Kyiv 03049, Ukraine

${ }^{4}$ State Enterprise "Kyiv Scientific Research Institute of Hydrodevices", Surikova Str., 3, Kyiv 03035, Ukraine
} 
due to the repeated exchange of sound waves emitted and reflected elements of the system $[1,6,7]$. In quantitative terms, the degree of acoustic interaction and its influence on the acoustic fields generated by the system is determined by many physical factors. Among them, in the electric field, we single out the nature of the electrical excitation of the radiators of the system; in a mechanical field, the mode composition of the oscillations of the radiators $[4,5,8]$, in the acoustic field - the physical and wave properties of the system and its elements [9-11].

In this case, we particularly note the fact that the acoustic field of the radiator, which is involved in the process of energy conversion in it, is the result of the acoustic interaction of all elements of the system.

It is the lack of until recently developed approaches that allow combining piezoceramic radiator $\mathrm{s}$ with a single radiation emission process, energy conversion processes in radiators and the formation of an acoustic field in an external space surrounding a system, were the main reason for the differences between the calculated and real values of field parameters of complex radiating systems. It should be noted that recently a number of works have appeared that demonstrate the search and development of new approaches to the solution of the formulated problem [3-8]. However, the abundance of physical factors affecting the processes of sound emission by complex electroacoustic systems suggests that these solutions are only the initial stage of the necessary research.

The purpose of this work is the further development of approaches to the determination of physical fields and their mutual influence on each other when sound is emitted by circular systems of cylindrical piezoceramic radiators with acoustically soft screens in the internal cavity of systems.

\section{Main part}

Consider the problem of sound emission by a circular cylindrical system (Fig. 1), formed from a finite number of cylindrical piezoceramic radiators (1) and a cylindrical acoustic screen (2) located in the internal cavity of the system.

The longitudinal axis of the radiators and the screen are parallel to each other. Each of the radiators is an extended sealed piezoceramic sheath, the internal cavity of which is evacuated or filled with air at normal atmospheric pressure or contains liquid or air at overpressure. Piezoceramic shells are circularly polarized. Electrically all the prisms forming the shell are rigidly glued, connected in parallel and an electrical signal of the form is connected to them $\psi_{S}=\psi_{0}^{S} e^{-i \omega t}$, where $S$ - current radiator number in the system, $\psi_{0}^{S}$ - voltage amplitude, $\omega$ - circular excitation of frequency. The acoustic screen is made in the form of an acoustically soft cylindrical shell.

In the most general form, the computational model of the system under consideration can be represented with the introduction of the following assumptions.

We will assume that the system is formed from $N+1$ elements, where $N$ - number of radiators, all elements of the system are infinitely long, and the properties of the radiators and the screen are homogeneous along their length. The system is placed in a liquid medium with density $\rho$ and sound velocity $c$. Inside each radiator there is a medium with density $\rho_{S}$ and speed 


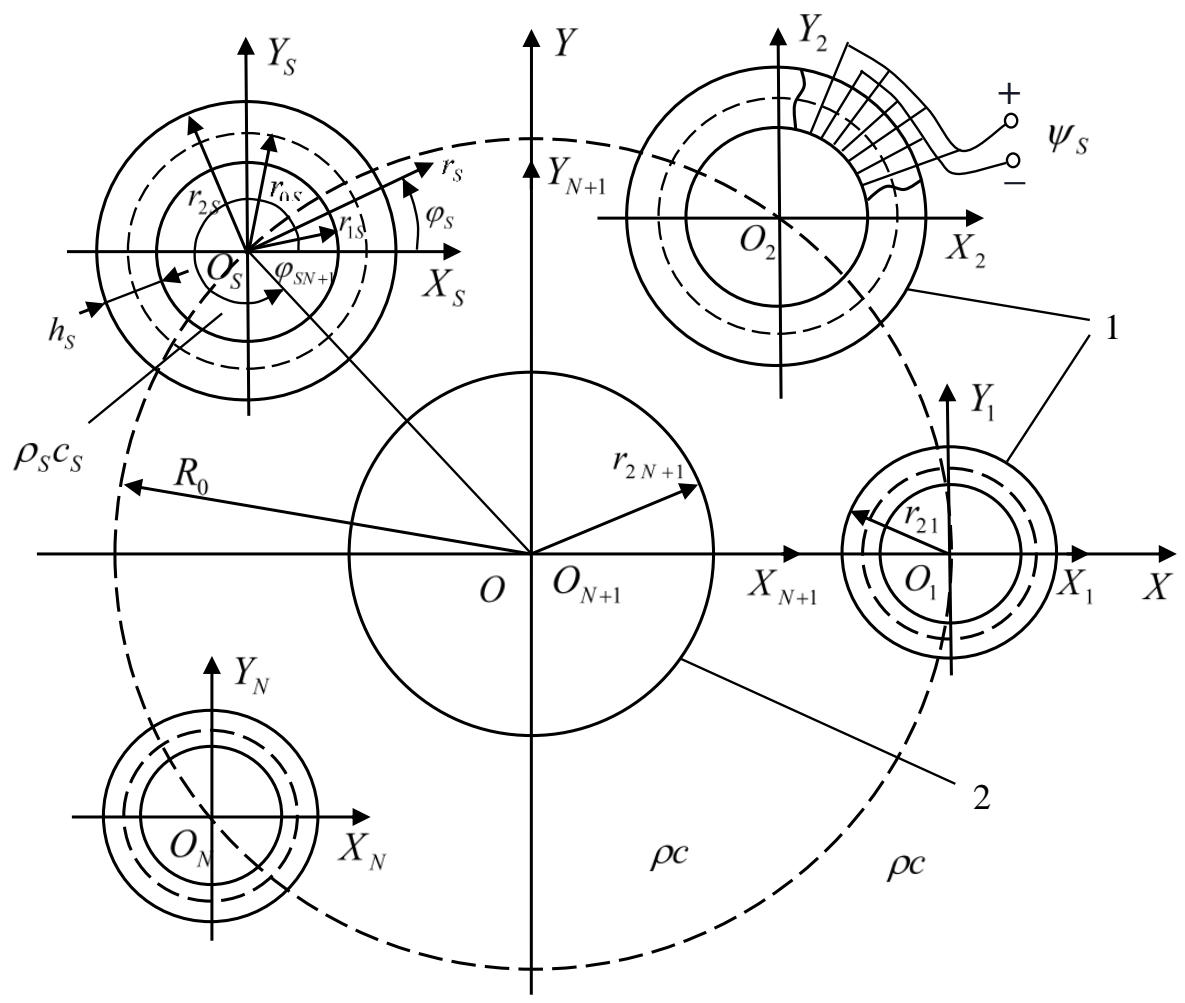

Fig. 1. Normal section of the cylindrical system of radiators with a screen

of sound $c_{S}$. In this case, the radiators themselves are thin piezoceramic shells, the circumferential polarization of which is created by the parallel connection $M_{S}$ of prisms rigidly glued to each other, on the side surfaces of which thin electrodes are applied.

In accordance with the given calculation model, to determine the physical fields of a circular cylindrical system with a screen, it is necessary to carry out a joint solution of such differential equations:

- Helmholtz equations describing the motion of elastic media (gas or liquid) inside and outside the elements of the system:

$$
\Delta \Phi^{(S)}+k_{s} \Phi^{(S)}=0 ; \quad S=1, \ldots, N+1 ;
$$

- equations of motion [2] of thin piezoceramic shells of radiators with circular polarization in displacements:

$$
\begin{gathered}
\left(1+\beta_{S}\right) \frac{\partial^{2} u^{(S)}}{\partial \varphi_{S}^{2}}+\frac{\partial w^{(S)}}{\partial \varphi_{S}}-\beta_{S} \frac{\partial^{3} w^{(S)}}{\partial \varphi_{S}^{3}}=a_{S} \gamma_{S} \frac{\partial^{2} u^{(S)}}{\partial t^{2}}, \\
-\frac{\partial u^{(S)}}{\partial \varphi_{S}}+\beta_{S}\left(\frac{\partial^{3} u^{(S)}}{\partial \varphi_{S}^{3}}-\frac{\partial^{4} w^{(S)}}{\partial \varphi_{S}^{4}}\right)-w^{(s)}+\frac{e_{33}^{(S)} r_{O S}}{C_{33 S}^{E(s)}} E_{\varphi}^{(S)} \\
+\frac{a_{S}}{h_{S}} q_{r}^{(S)}=a_{S} \gamma_{S} \frac{\partial^{2} w^{(S)}}{\partial t^{2}} ; \quad S=1, \ldots, N ;
\end{gathered}
$$


- equations of forced electrostatics for piezoelectric ceramics of the $S$-th radiator:

$$
\vec{E}_{S}=-\operatorname{grad} \psi_{S} ; \quad \operatorname{div} \vec{D}_{S}=0 ; \quad S=1, \ldots, N
$$

In expressions (1)-(3) accepted: $\Delta$ - the Laplasian operator; $S$ - acoustic potential of the $S$-th radiator; $k$ and $k_{2 S}$ - wave numbers of media outside and inside the $S$-th radiator; $u^{(S)}$ and $w^{(S)}$ - tangent and normal components of the displacement vector of points of the middle surface of the $S$-th radiator;

$$
\eta_{S}=\frac{h_{S}^{2}}{12 r_{0 S}^{2}}\left(1+e_{33}^{2(S)} / C_{33}^{E(S)} \varepsilon_{33}^{S(S)}\right) ; \quad a_{S}=r_{O S}^{2} / C_{33}^{E(S)}
$$

$q_{r S}$ - external load of the $S$-th radiator; $C_{33}^{E(S)}, \varepsilon_{33}^{S(S)}, e_{33}^{(S)}, \gamma_{S}$ - respectively, the modulus of elasticity at zero electric intensity, the dielectric constant at zero deformation, the piezoelectric constant and the density of the material of the piezoceramic shell of the $S$-th radiator, $\vec{E}^{(S)}$ and $\vec{D}^{(S)}$ - vectors of intensity and induction of the electric field of the $S$-th radiator.

The region of existence of the sound field is multiply connected, since the sound fields of the radiators are formed in two areas - the external $\Phi_{1}^{(S)}$ and internal $\Phi_{2}^{(S)}$, and the acoustic field of the acoustic screen $\Phi_{1}^{(N+1)}$ - only in the outer area. The boundaries of areas are determined by the relations: for internal areas

$$
0 \leq\left|\varphi_{S}\right| \leq \pi, \quad 0 \leq r_{S} \leq r_{1 S}=r_{0 S}-h_{S} / 2, \quad S=1, \ldots, N
$$

for external areas

$$
\begin{gathered}
0 \leq\left|\varphi_{S}\right| \leq \pi, \quad r_{S} \geq r_{2 S}=r_{0 S}+h_{S} / 2, \quad S=1, \ldots, N \\
r_{N+1}>r_{2, N+1} .
\end{gathered}
$$

Taking into account the above boundaries, the kinematic and dynamic conditions of conjugation of sound fields on these boundaries take the form:

- on the surfaces of each radiator, the conditions of equality of the velocities of vibration of the particles of the media and the velocities of the normal displacements of the surfaces of the radiators are satisfied:

$$
\begin{gathered}
-\frac{\partial \Phi_{1}\left(r_{S}, \varphi_{S}\right)}{\partial r_{S}}=\frac{\partial w^{(S)}}{\partial t}, \quad 0 \leq\left|\varphi_{S}\right| \leq \pi, r_{S}=r_{2 S}, S=1, \ldots, N, \\
-\frac{\partial \Phi_{2}^{(S)}\left(r_{S}, \varphi_{S}\right)}{\partial r_{S}}=\frac{\partial w^{(S)}}{\partial t}, \quad 0 \leq\left|\varphi_{S}\right| \leq \pi, r_{S}=r_{1 S}, S=1, \ldots, N,
\end{gathered}
$$

where $\Phi_{1}\left(r_{S}, \varphi_{S}\right)$ - the radiation field of the system, expressed in the coordinates of the $S$-th radiator;

- on the surface of each radiator, the condition of equality of the normal component of the stress tensor $\sigma_{r}^{S}$ sound pressure taken with the opposite sign:

$$
\sigma_{r}=q_{r}=-\left(p_{1}-p_{2}^{(S)}\right), \quad 0 \leq\left|\varphi_{S}\right| \leq \pi, r_{S}=r_{0 S}, S=1, \ldots, N,
$$



where $p_{1}=\rho \frac{\partial \Phi_{1}}{\partial t}$ and $p_{2}^{(S)}=\rho_{S} \frac{\partial \Phi_{2}^{(S)}}{\partial t}$-radiation loads from dynamic pres-
sures;

- on the surface of an acoustically soft screen, the condition that the sound pressure is zero:

$$
\Phi_{1}\left(r_{N+1}, \varphi_{N+1}\right)=0, \quad 0 \leq\left|\varphi_{N+1}\right| \leq \pi, \quad r_{N+1}=r_{2, N+1} .
$$

Electrical boundary conditions taking into account the dynamic deformation of the $S$-th cylindrical radiator voltage $\psi_{S}=\psi_{0}^{S} e^{-i \omega t}$ and equations (3) take the form [2]:

- the electric field strength in the material of the piezoceramic shell of the $S$-th radiator with a circumferential polarization:

$$
E_{\varphi}^{(S)}=-\frac{\psi_{0}^{(S)} M_{S}}{2 \pi r_{0 S}}, \quad S=1, \ldots, N
$$

- components of electrical induction along the radius $D_{r}^{(S)}$, longitudinal axis $D_{z}^{(S)}$ and circumference $D_{\varphi}^{(S)}$ equal respectively:

$$
\begin{gathered}
D_{r}^{(S)}=0 ; \quad D_{z}^{(S)}=0 ; \\
D_{\varphi}^{(S)}=\varepsilon_{33}^{S(S)} E_{\varphi}^{(S)}+e_{33}^{(S)}\left(\frac{1}{r} \frac{\partial u^{(S)}}{\partial \varphi_{S}}+\frac{w^{(S)}}{r_{(S)}}\right) .
\end{gathered}
$$

These conditions are complemented by radiation conditions at infinity and the absence of features in the internal cavities of each of the radiators of the system.

To solve the formulated problem, we use the method of coupled fields in multiply connected regions. Imagine the displacement of the shells of the radiators, describing the mechanical fields of the radiators, in the form of expansions $u^{(S)}$ and $w^{(S)}$ on its own forms of oscillations of shells in vacuum

$$
\begin{aligned}
u^{(S)} & =\sum_{n} u_{n}^{(S)} e^{i n \varphi_{S}} ; \\
w^{(S)} & =\sum_{n} w_{n}^{(S)} e^{i n \varphi_{s}} ;
\end{aligned}
$$

Coefficients $u^{(S)}$ and $w^{(S)}$ allow to take into account the interaction of acoustic, mechanical and electric fields in the process of conversion of electrical energy into mechanical and acoustic.

Acoustic fields of the system that satisfy the Helmholtz equation (1) are described as follows.

The acoustic field of the system in its environment will be represented as a superposition of the fields $\Phi_{1}^{(S)}$ created by each element of the system:

$$
\Phi_{1}=\sum_{S=1}^{N+1} \Phi_{1}^{(S)}
$$


In this case, all the fields $\Phi_{1}^{(S)}$ should be determined taking into account the interaction of all elements of the system with each other by the acoustic field resulting from the multiple scattering of waves on all elements of the system. Acoustic fields created by radiators and a screen in the external environment are represented by expansions in wave cylindrical functions satisfying the radiation condition at infinity:

$$
\Phi_{1}^{(S)}\left(r_{S}, \varphi_{S}\right)=\sum_{n} A_{n}^{(S)} H_{n}^{(1)}\left(k r_{S}\right) e^{i n \beta \varphi_{S}}, \quad S=1, \ldots, N+1
$$

Acoustic fields arising from the radiation of sound in the internal regions of the radiators and having no features in them can be described by the expressions:

$$
\Phi_{2}^{(S)}\left(r_{S}, \varphi_{S}\right)=\sum_{n} B_{n}^{(S)} J_{n}\left(k_{2 S} r_{S}\right) e^{i n \varphi_{S}}, \quad S=1, \ldots, N+1
$$

In relations (12) and (13), the traditional designations of cylindrical functions are used; through coefficients $A_{n}^{(S)}$ interaction of elements of the system by the acoustic field due to multiple scattering of waves is taken into account; through coefficients $B_{n}^{(S)}$ interaction of acoustic fields in the internal areas of their radiators is taken into account.

For determining the coefficients of decomposition $u_{n}^{(S)}, w_{n}^{(S)}, A_{n}^{(S)}$ and $B_{n}^{(S)}$ it is necessary to use relations (2), (4)-(7). Substitution of formulas (10) into expression (2) allows us to express $u_{n}^{(S)}$ through $w_{n}^{(S)}$ in the following form:

$$
u_{n}^{(S)}=\frac{i\left(n+\beta_{S} n^{3}\right)}{\left(1+\beta_{S}\right) n^{2}-\omega^{2} \alpha_{S} \gamma_{S}} w_{n}^{(S)} .
$$

Since the entered into the expression (11) fields are $\Phi_{1}^{(S)}$ specified in their local coordinate systems, relations (11) and (12) cannot be substituted into the boundary conditions (4)-(7) and equation (1) and require the representation in local coordinates of that system element whose boundary conditions are used. The transfer of coordinate systems is provided using the addition theorems for cylindrical wave functions [1]:

$$
\left.H_{m}^{(1)}\left(k r_{q}\right) e^{i m \varphi_{q}}=\sum_{n} J_{n}\left(k r_{S}\right) H_{m-n}^{(1)}\right)\left(k r_{q S}\right) e^{i(m-n) \varphi_{q S}} e^{i n \varphi_{S}}
$$

where $r_{q S}$ and $\varphi_{q S}$ - polar coordinates of the origin of the coordinate system $O_{S}$ in the coordinates of the $q$-th system.

Algebraization of systems of functional equations (1)-(7) using relations (8), (10)-(15) and the properties of completeness and orthogonality of systems of angular functions on the interval $[0,2 \pi]$ allows you to get to determine the unknown coefficients of expansions $u_{n}^{(S)}, w_{n}^{(S)}, A_{n}^{(S)}$ and $B_{n}^{(S)}$ infinite system of linear algebraic equations of the form: 


$$
\begin{aligned}
& -B_{n}^{(S)} J^{\prime}\left(k_{2 S} r_{1 S}\right)+i c_{2 S} w_{n}^{(S)}=0, \\
& S=1, \ldots, N, n=-\infty, 0, \infty ; \\
& i c w_{n}^{(S)}-\left[A_{n}^{(S)} H_{n}^{(1)^{\prime}}\left(k r_{2 S}\right)\right. \\
& \left.+\sum_{\substack{q=1 \\
q \neq S}}^{N+1} \sum_{m} A_{m}^{(q)} J_{n}^{\prime}\left(k r_{2 S}\right) H_{m-n}^{(1)}\left(k r_{q S}\right) e^{i(m-n) \varphi_{q S}}\right]=0 \\
& S=1, \ldots N \\
& A_{n}^{(N+1)} H_{n}^{(1)}\left(k r_{2 N+1}\right) \\
& \begin{aligned}
+\sum_{q=1}^{N} \sum_{m} A_{m}^{(q)} J_{n}^{\prime}\left(k r_{2 N+1}\right) H_{m-n}^{(1)} e^{i(m-n) \varphi_{q N+1}} & =0, \\
n & =-\infty, 0, \infty
\end{aligned} \\
& R_{n}^{(S)} w_{n}^{(S)}+\frac{\alpha_{S}}{h_{S}} i \omega \rho\left[A_{n}^{(S)} H_{n}^{(1)}\left(k r_{q S}\right)\right. \\
& \left.+\sum_{\substack{q=1 \\
q \neq S}}^{N+1} \sum_{m} A_{m}^{(q)} J_{n}\left(k r_{2 S}\right) H_{m-n}^{(1)}\left(k r_{q S}\right) e^{i(m-n) \varphi_{q S}}\right] \\
& -\frac{\alpha_{S}}{h_{S}} i \omega \rho_{S} B_{n}^{(S)} J_{n}\left(k_{2 S} r_{1 S}\right) \\
& =-\frac{e_{33}^{(S)}}{C_{33}^{E(S)}} \frac{M_{S} \psi_{0}^{(S)}}{2 \pi} \int_{0}^{2 \pi} e^{i n \varphi_{S}} d \varphi_{S}, \\
& S=1, \ldots, N, n=-\infty, 0, \infty, \quad
\end{aligned}
$$

where $R_{n}^{(S)}=n^{2}\left(1+\beta_{S} n^{2}\right)^{2}-\left(1+\beta_{S} n^{4}-\alpha_{S} \gamma_{S} \omega^{2}\right)\left(n^{2}+\beta_{S} n^{2}-\alpha_{S} \gamma_{S} \omega^{2}\right) /(1+$ $\left.\beta_{S}\right) n^{2}-\alpha_{S} \gamma_{S} \omega^{2}$, the prime means the derivative of the function.

Let us define expressions that allow quantitative estimates of the magnitude of the electric current flowing in the excitation circuit of the $S$-th radiator of a circular system of radiators with a screen. From physical considerations it is clear that the placement of cylindrical radiators in such a system (Fig. 1) violates the radial symmetry of their radiation load and, as a result, the radial symmetry of mechanical vibrations of the piezoceramic shells of radiators. Therefore, the magnitude of the excitation current in the circuit of each of $M_{S}$ parallel connected piezoceramic prisms of the $S$-th radiator will be tied to the angular position of these prisms in the radiator shell. Then the expression for the total current $I^{(S)}$ in the excitation circuit of the $S$-th emitter of the system under consideration takes the form:

$$
I^{(S)}=S_{e l}^{S} \sum_{j=1}^{M_{S}} \frac{\partial D_{\varphi_{S}}^{(j)}}{\partial t},
$$


where $S_{e l}^{S}$ - the area of the electrode deposited on the flat surface of the piezoceramic prism of the $S$-th radiator.

Having executed a number of transformations, expression (17) with regard to relations (8)-(10) can be represented as:

$$
\begin{aligned}
I^{(S)}= & -i \omega S_{e l}^{S}\left\{-\varepsilon_{33}^{S(\mathrm{~S})} \frac{\psi_{0 S} M_{S}^{2}}{2 \pi r_{0 S}}\right. \\
& +\frac{e_{33}^{(S)}}{r_{0 S}} \sum_{j=1}^{M_{S}}\left[\sum_{n} i n u_{n}^{(S)} e^{i n \frac{2 n j}{M_{S}}}+\sum_{n} w_{n}^{(S)} e^{\left.\left.i n \frac{2 n j}{M_{S}}\right]\right\} .}\right.
\end{aligned}
$$

Analysis of the relation (18) shows that the total electric current in the excitation circuit of each $S$-th piezoceramic radiator $(S=1, \ldots, N)$ of the circular system of radiators with a screen is the sum of two components of the current - capacitive and dynamic.

The obtained expressions allow you to perform quantitative estimates of the parameters of the electric, mechanical and acoustic fields of the radiators when operating in circular systems with a screen and, based on the analysis of the results obtained, identify the degree of influence of the fields on each other.

As it was already mentioned, in radiating systems based on piezoceramic radiators, the radiation process is determined by the mutual coupling of electric, mechanical and acoustic fields. Let us analyze these relations and estimate the priorities of their influence in the formation of the physical fields under consideration. To do this, we use the results of a numerical experiment to estimate the frequency and angular dependences of the amplitudes and phases of the acoustic, mechanical, and electric fields of the radiators as part of the systems under consideration. Calculations were made according to the above formulas for radiating systems with parameters:

$$
\begin{aligned}
& N=3 ; r_{0}=0,068 \mathrm{~m} ; h=0,008 \mathrm{~m} ; \\
& M_{s}=48 ; \\
& e_{33}^{(S)}=14,9 \mathrm{C} / \mathrm{m}^{2} ; \\
& \varepsilon_{33}^{(S)}=1280 \cdot 8,85 \cdot 10^{-12} \mathrm{~F} / \mathrm{m} ; \\
& \gamma_{S}=7210 \mathrm{~kg} / \mathrm{m}^{3} ; \\
& C_{33}^{(S)}=13,6 \cdot 10^{-10} \mathrm{~N} / \mathrm{m}^{2} ; \\
& \rho=10^{3} \mathrm{~kg} / \mathrm{m}^{3} ; \\
& c=1,5 \cdot 10^{3} \mathrm{~m} / \mathrm{s} ; \\
& r_{2 N+1}=0,072 \mathrm{~m} ; \\
& R_{0}=r_{2 N+1}+r_{0}+5 \mathrm{~mm} ; \\
& \rho_{S} c_{S}=0 \text { for all } S=1, \ldots, N . \\
&
\end{aligned}
$$

The frequency characteristics of the amplitudes and phases of the fields were calculated on the surface of the radiators at points opposite the screen from the outside of the system.

The analysis of the above analytical relations allows to establishing three important physical features that determine the interaction of the fields of the radiating circular system with the screen. The first feature is connected with the interaction of the electric and mechanical fields of the radiators and consists in the fact that with the chosen method of electrical loading of circular cylindrical piezoceramic radiators their electric field is radially symmetric. Therefore, 
in the mechanical field of a circular piezoceramic radiator, such an electric field can excite only one zero own mode of oscillation and "pump" energy into it only in this zero mode [4]. This can be seen from the fourth expression of the system of equations (16), where the right-hand side describing the electric field of the radiator is related to its mechanical field and is not zero only on the zero ( $\mathrm{n}=0)$ mode of oscillation of the $S$-th radiator. Thus, the relationship between the electric and mechanical fields in a cylindrical piezoceramic transducer seems relatively simple.

The second feature is associated with the interaction of the acoustic fields of all elements of the radiating system, due to the repeated exchange between them of the radiated and reflected waves. The degree of this interaction, expressed by the double sum in relations (16), and the level of its influence on the generated fields is determined in the expressions of system (16) by the value of the multiplier $H_{m-n}^{(1)}\left(k r_{q S}\right)$, which describes the interaction between the $m$-th and $n$-th order acoustic waves created by the $q$-th and $S$-th elements of the system $(q=1, \ldots, N+1 ; S=1, \ldots, N+1)$, that are spaced over a distance $r_{q S}$.

The third feature characterizes the mutual influence of the acoustic and mechanical fields of the radiators within the system under study and the dependence of this influence on the acoustic interaction of its elements. The physical consequence of the acoustic interaction of elements of a circular system with a screen is a violation of the radial symmetry of the acoustic radiation loading of its radiators. Indeed the form of expressions analysis (16), within $H_{m-n}^{(1)}\left(k r_{q S}\right) \rightarrow 0$ the acoustic interaction disappears and when the cylindrical radiators of the system at the zero mode of their mechanical vibrations are electrically excited, the acoustic fields they generate have radial symmetry. This indicates that in the absence of acoustic interaction of the elements of the system, the connection between the acoustic and mechanical fields is characterized by the same relative simplicity as the connection between the electric and mechanical fields of the radiators of the system. The occurrence of the acoustic interaction of elements in the system under study fundamentally changes the situation. It is thanks to him that the radial symmetry of the acoustic loading of each radiator of a circular system with a screen is broken. In the mechanical field of the radiator with broken symmetry appear, as it follows from the analysis of the system of equations (16), the oscillations that follow the zero mode. This means that the energy of the electric field, "pumped" into the mechanical field of the radiator only at the zero mode of its oscillations, is redistributed between all forms of oscillations. Thus, due to the emergence in the system of interaction of the acoustic fields of its elements, single-mode cylindrical piezoceramic radiators as part of the system acquire the property of multi-mode.

Let's confirm now the results of the presented theoretical reasoning by analyzing specific numerical data.

An analysis of the angular dependences of the amplitudes of the acoustic field on the surfaces of the radiators of a circular system with a screen (Fig. 2) suggests that the occurrence of interaction of the system elements in the external environment over the acoustic field is the cause of the considerable heterogeneity of the amplitude distribution under study. 

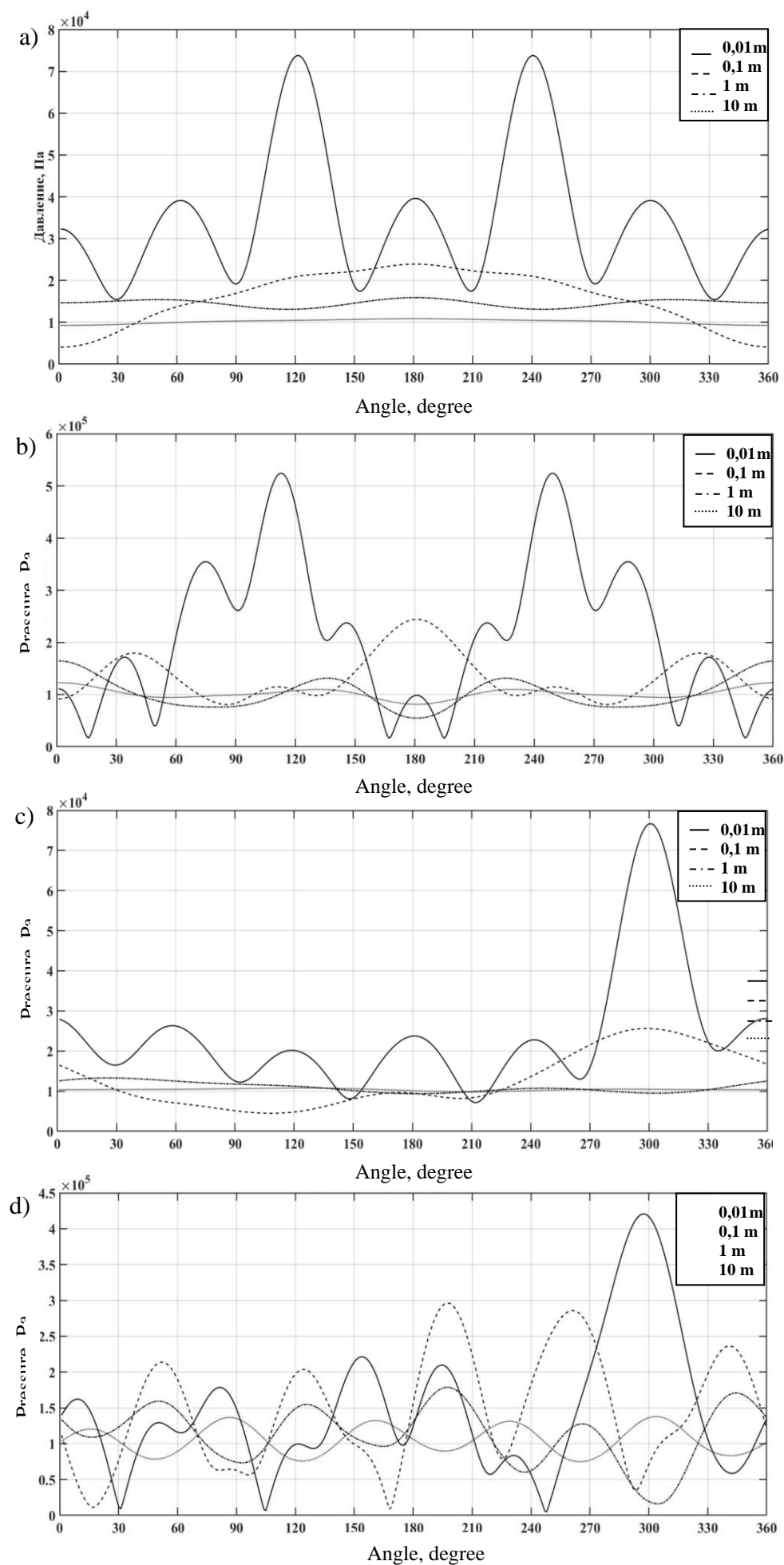

Fig. 2. Angular dependences of the amplitudes of the acoustic pressure of the average $(\mathrm{a}, \mathrm{b})$ and extreme $(\mathrm{c}, \mathrm{d})$ radiators of a circular system with a screen with sector sound emission at different frequencies of $2890 \mathrm{~Hz}$ (a), $9500 \mathrm{~Hz}$ (b), $2890 \mathrm{~Hz}$ (c), $9940 \mathrm{~Hz}(\mathrm{~d})$, for different distances $l$ between system elements: $1-l=10^{-2} \mathrm{~m} ; 2-l=10^{-1} \mathrm{~m} ; 3-l=1 \mathrm{~m} ; 4-l=10 ; \mathrm{m}$. 
If the acoustic interaction was absent, then all radiators of the system would have a uniform pressure distribution on their surfaces. The degree of violation of the radial symmetry of the acoustic loading depends on the location of the radiator in the system and on the wave distance between its elements.

While maintaining radial symmetry of electrical loading of piezoceramic cylindrical radiators in a circular system with a screen, a natural consequence of the violation of the radial symmetry of their acoustic loading is the appearance of radiators in mechanical fields other than zero subsequent vibration modes (Fig. 3).
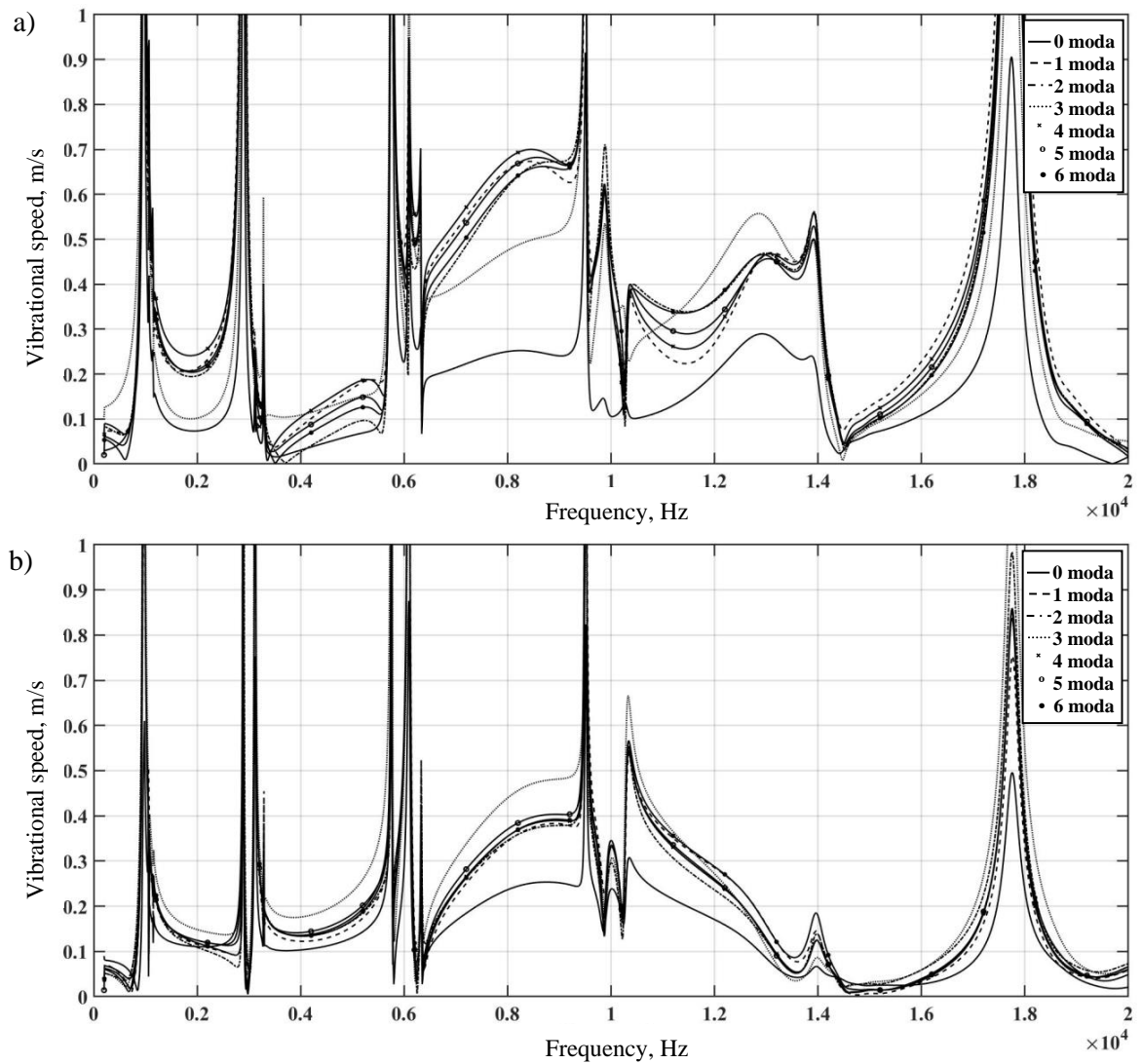

Fig. 3. Frequency dependences of the amplitudes of the modes of the oscillatory velocity on the surface of the average (a) and extreme (b) radiators that work as part of the system when the distance between the surfaces of the elements $l=10^{-2} \mathrm{~m}$.

At the same time, at individual frequencies, the amplitudes of the vibrational speeds of the newly generated modes are comparable or even exceed the amplitude of the speed of the zero mode, which is evidence of the effective redistribution in the mechanical field of the energy of the zero mode between the newly generated modes. When the distance between the elements of the circular system changes, the ratio between the amplitudes of the vibrational speeds of the zero and subsequent modes changes, increasing for the zero mode with increasing $k R_{0}$. 

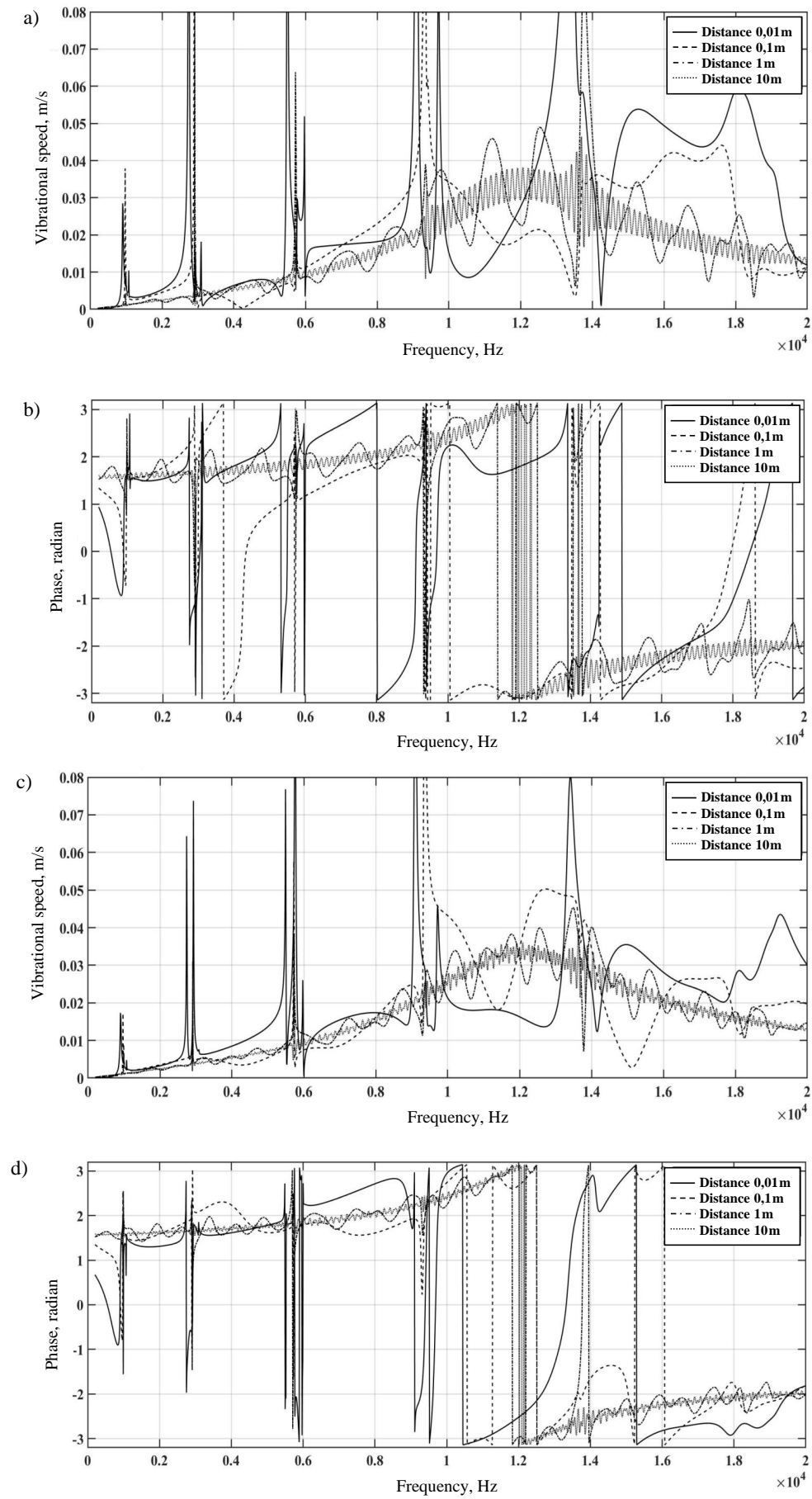

Fig. 4. Frequency dependences of the amplitudes (a, c) and phases (b, d) of the vibrational speeds of the average $(\mathrm{a}, \mathrm{b})$ and extreme $(\mathrm{c}, \mathrm{d})$ radiators of a circular system with a screen for different distances between the surfaces of the system elements during sector radiation. 
The generation of new vibration modes with their own vibrational speeds, which is the result of the interaction of the acoustic fields of the elements of a circular system with a screen in the external space of the system, significantly changes the frequency characteristics of its radiators. The decisive influence of the acoustic interaction on the mechanical fields of the radiators in the composition of the system is shown by the curves of Fig. 4. Their analysis shows that it is the interaction of the system elements in its external acoustic field, the magnitude and degree of influence of which depend on the distance between the elements, determines the regularities of the behavior of the frequency dependences of the amplitudes and phases of the parameters of mechanical fields. As the distance increases, the forms of the frequency dependences, the frequencies of the newly generated own mechanical resonances of the system and the amplitudes of their vibrational speeds change. At large distances, multiple exchanges of radiated and reflected waves between elements of the system weakens, the frequency characteristics of all radiators of the system become the same and take on the characteristics of the radiator when it is operated outside the system.

The interrelation between the mechanical and acoustic fields of the radiators of the system in the process of conversion of electric energy into acoustic energy is manifested both in the change of the mechanical fields of the radiators under the influence of the acoustic field of the circular system with the screen, and in the reverse effect of the modified mechanical fields on the acoustic fields of these systems, as shown by the graphs Fig. 5. Their analysis shows that each mode of the mechanical field of the radiator as part of a circular system with a screen forms its component of the acoustic field in the external space of the system (Fig. 5b, Fig. 5c), and the full field of the system (Fig. 5a) is the result of the superposition of the constituent elements of all elements of the system with their amplitudes and phases. Due to this, a number of features of the full acoustic field of a circular system arise, which consist in enriching the spectrum of the natural frequencies of the system and expanding this spectrum to low frequencies. In this case, in a circular system with a screen, self-resonant frequencies appear, 5-10 times lower than the frequencies of the mechanical resonance of the piezoceramic shells of the system radiators.

In conclusion, it is interesting to quantify the influence of all the abovementioned changes in the acoustic and mechanical fields of the system and its radiators on the electric fields that excite the radiators of the system. Curve analysis Fig. 6 and comparing them with the graphs of Fig. 7 shows that the earlier conclusion that the energy of the electric field is "pumped" into the mechanical field of the radiator with the chosen method of electrically loading it only at the zero mode of its oscillations is retained. However, the electric field of the radiator (Fig. 6) tracks all the subtleties that its mechanical field (Fig. 7) acquired under the influence of the acoustic field of a circular system with a screen. 

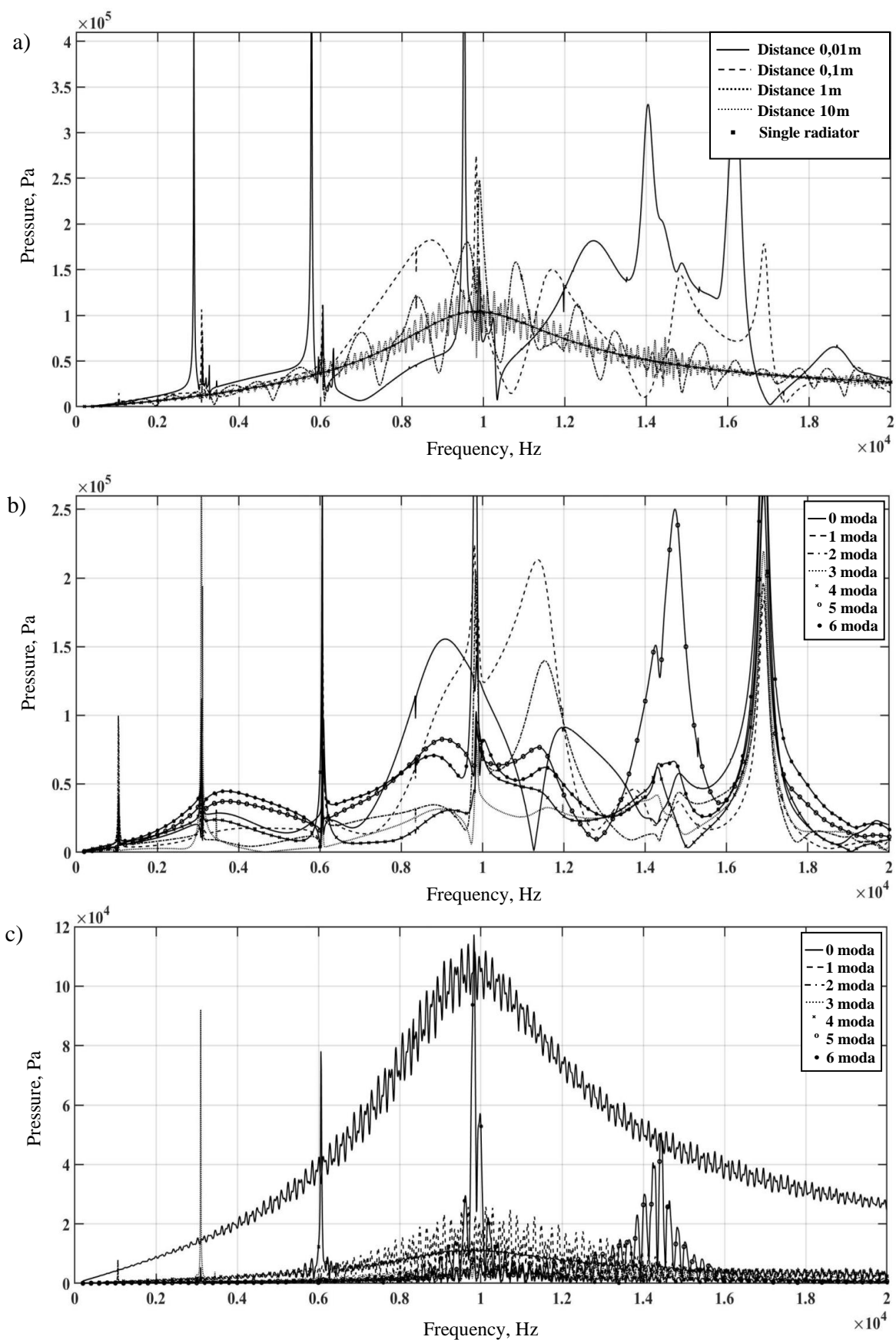

Fig. 5. Frequency dependences of the amplitudes of the total (a) acoustic pressure and its modal components of the average radiators with sector radiation of circular systems with a screen at different distances $l$ between the surfaces of the system elements: $l=0,1 \mathrm{~m}(\mathrm{~b})$;

$$
l=10 \mathrm{~m}(\mathrm{c})
$$


a)

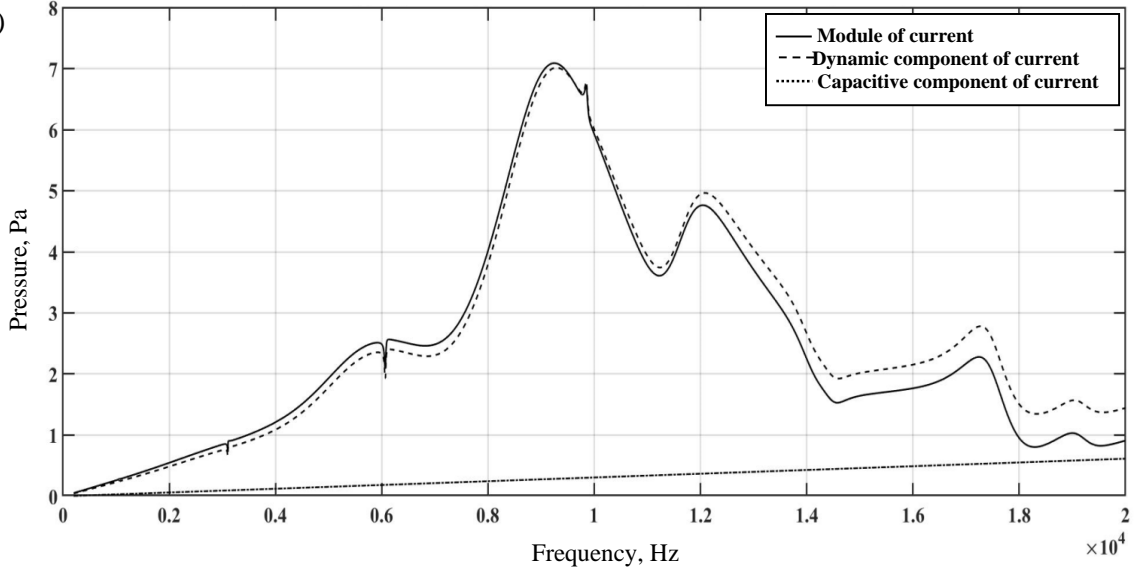

b)

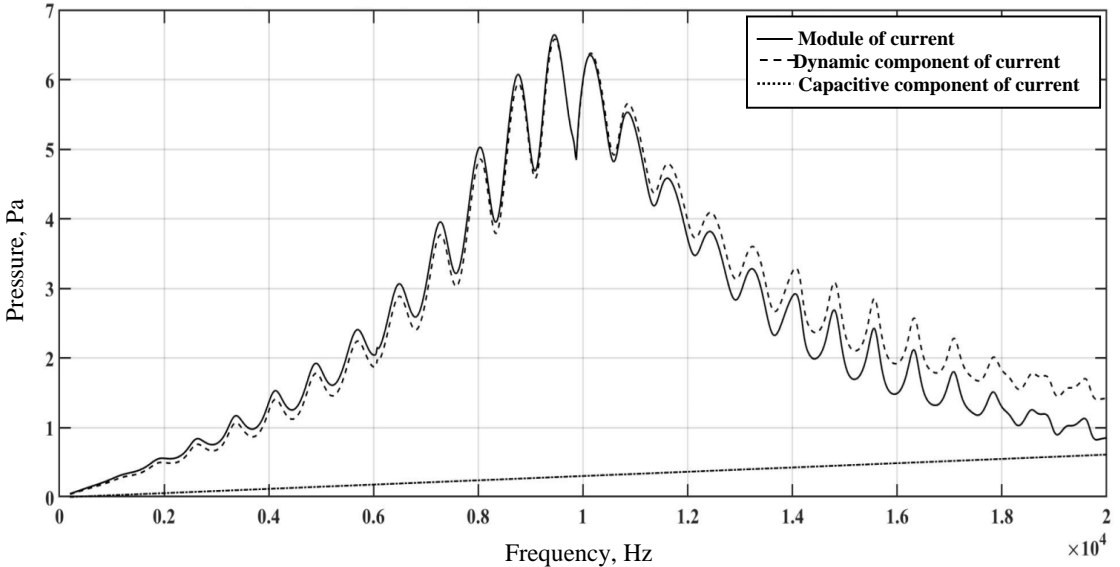

c)

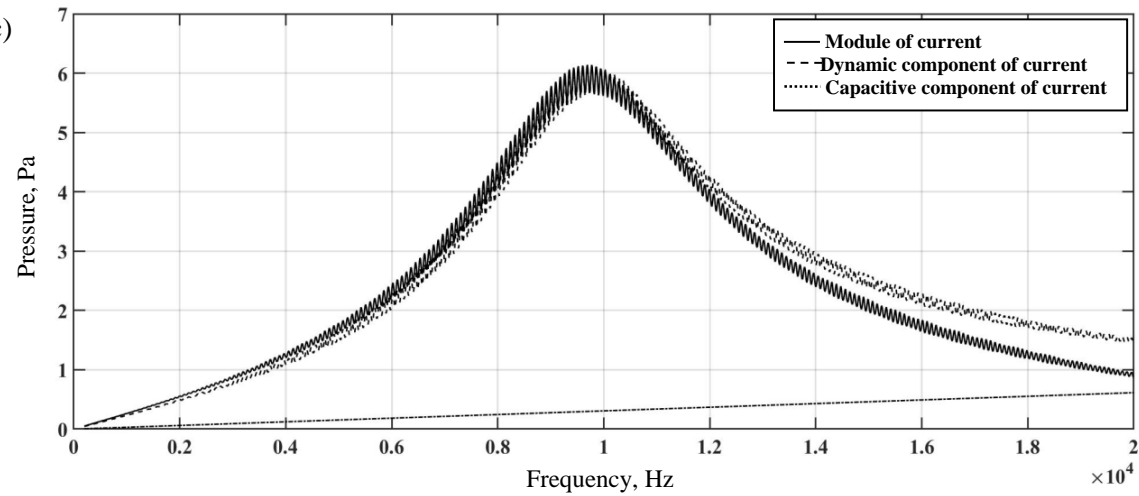

Fig. 6. Frequency dependences of the amplitudes of the electric current in the external circuits of the radiators of a system with a screen with circular radiation and distances between the surfaces of the screen $r_{2 N+1}$ and radiators $r_{2 S}$, equal to $0.1 \mathrm{~m}$ (a), $1 \mathrm{~m}$ (b), $10 \mathrm{~m}(\mathrm{c})$ 
a)
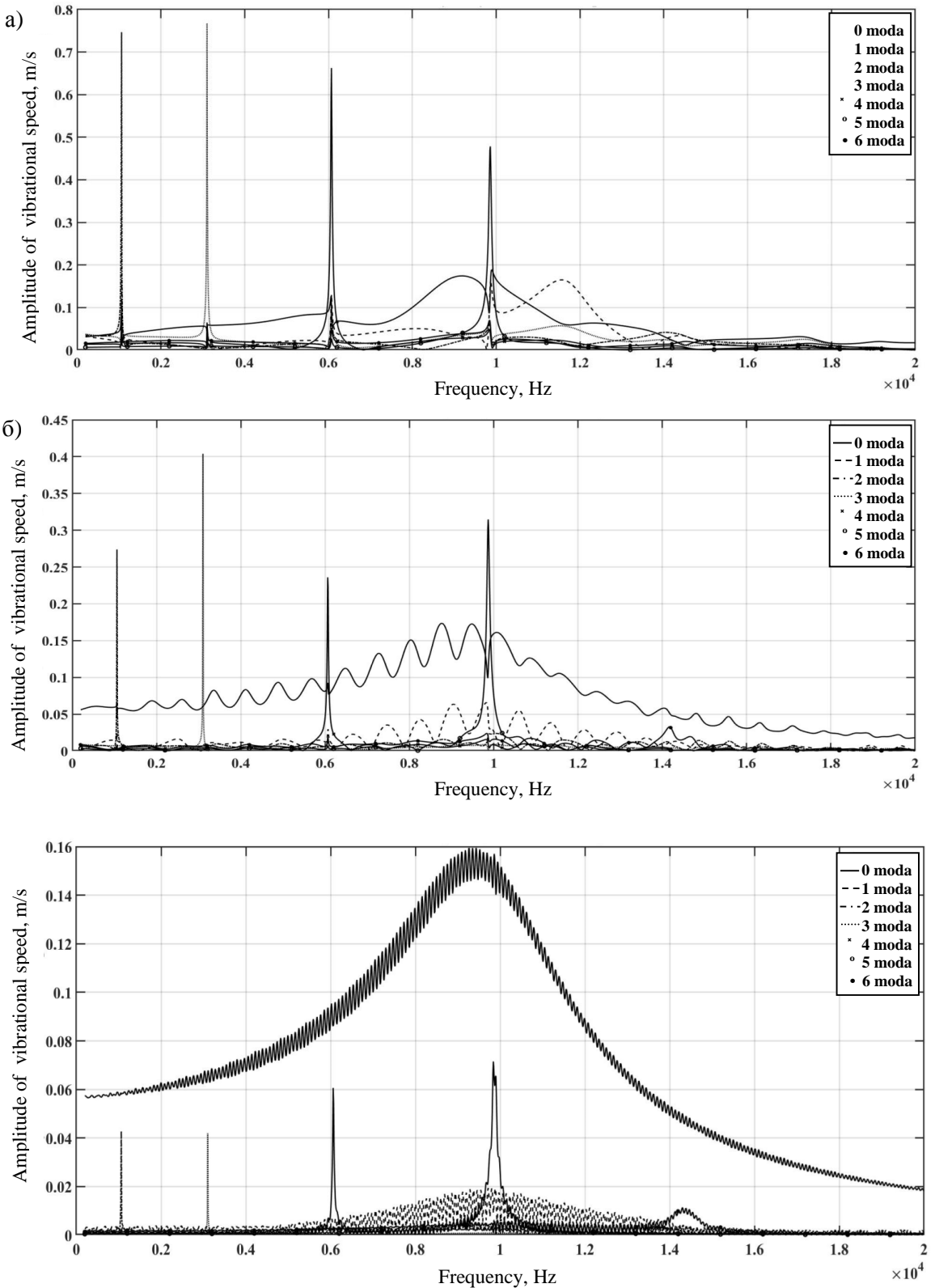

Fig. 7. Frequency dependences of the amplitudes of the modes of the vibrational speed of the radiators of a system with a screen with circular radiation and distances between the surfaces of the screen $r_{2 N+1}$ and radiators $r_{2 S}$, equal to $0.1 \mathrm{~m}$ (a), $1 \mathrm{~m}$ (b), $10 \mathrm{~m}$ (c). 


\section{Conclusion}

The process of radiation of sound by circular systems with a screen formed from cylindrical piezoceramic radiators is characterized by two functional features, namely, the conversion of electrical energy into mechanical energy, and the latter into acoustic energy and the formation of acoustic energy in spaces surrounding the system. In this case, a number of interactions of the indicated physical fields arise. When converting electrical energy into acoustic, the electric, mechanical and acoustic fields interact. During the formation of the acoustic field of the system in the surrounding spaces, the interaction of the acoustic fields of its elements occurs, due to the multiple exchange of radiated and reflected waves between its elements. With the radial symmetry of the exciting radiator of the electric field, its interaction with the mechanical field of a cylindrical piezoceramic radiator occurs only at the zero mode of its mechanical oscillations. It has been established that the interaction of the mechanical fields of the radiators with the acoustic field of the system is a much more complex process. In this process, the decisive role is played by the interaction of the acoustic fields of the system elements in the external space. It is thanks to this interaction that the radial symmetry of the acoustic loading of a single cylindrical radiator is broken during its operation as part of the system, leading to the multimode radiators. Analytical relationships have been obtained and, on their basis, by a method of numerical experiment, qualitative and quantitative laws have been revealed on the influence of the studied interactions on the radiation of sound by circular systems with screen.

\section{References}

[1] Leiko A.G., Yu.E. Shamarin and V.P. Tkachenko. 2000. Underwater acoustic equipment and devices: in 2 volumes. T.1. Underwater acoustic antennas. Methods for calculating sound fields. Kyiv, Ukraine: Avanpostprim. [in Russian]

[2] Grinchenko V.T., A.F. Ulitko and N.A. Shulga. 1989. Mechanics of coupled fields in structural elements: T. 5. Electroelasticity. Kyiv, Ukraine: Naukova Dumka. [in Russian]

[3] Nyzhnik O.I. 2018. Radiation of hydroacoustic signals by planar antenna arrays generated from cylindrical piezoceramic converters. Diss. for the sciences Art. Ph.D. Kyiv, Ukraine. [in Ukrainian]

[4] Grinchenko V.T., I.V. Vovk and V.T. Matsypura. 2013. Acoustics wave problems. Kyiv, Interservice. [in Russian]

[5] Korzhik O.V. 2011. Hydroacoustic devices based on multimode piezoceramic converters. Diss. for the sciences Art. D.Sc. Kyiv, Ukraine. [in Ukrainian]

[6] Derepa A.V. 2015. Hydroacoustic stations with towed antennas of concentrated type and ways of creating them the direction of a special form. Journal Armament and military equipment. Central research institute of armament and military equipment of the Armed Forces of Ukraine. 4(8):9-14. 
[7] Derepa A.V. 2015. Ways of increasing the efficiency of the system "hydroacoustic station - a surface ship" with lumped antennas of variable depth. Journal Electronics and communication. National Technical University of Ukraine "Igor Sikorsky Kyiv Polytechnic Institute". 20(6):51-58.

[8] Leiko A.G., A.I. Nizhnik and Ya.I. Starovoit. 2013. Acoustic properties of cylindrical piezoceramic radiators of a power and compensated design with a longitudinal and transverse piezoelectric effect. Journal Electronics and communication. National Technical University of Ukraine "Kiev Polytechnic Institute named after Igor Sikorsky". 6:62-73.

[9] Ivanov E.A. 1968. Diffraction of electromagnetic waves on two bodies. Minsk, USSR: Science and Technology. [in Russian]

[10] Aronov B.S. 2009. Couplend vibration analysis of the thinwalled cylindrical piezoelectric ceramic transducers. The Journal of the Acoustical Society of America. 12(3):803-818.

[11] Aronov B.S., A.B. David and L.B. Corey. 2007. Effects of coupled vibrations on the acoustical performance of underwater cylindrical shell transducers. The Journal of the Acoustical Society of America. 122(2):3419-3428.

[12] Jin O.K., G.L. Jung and G.J. Hyung. 2004. Radial vibration characteristics of piezoelectric cylindrical transducers. Journal of Sound and vibration. 300(1-2):241-249. 